\title{
ON THE THEORY OF NON-HOLONOMIC SYSTEMS IN THE FINSLER SPACE
}

\author{
Yoshie Katsurada \\ (Received November 1, 1949; in revised form, November 30, 1950)
}

Introduction. G. Vranceanu ${ }^{1)}$ introduced the concept of a non-holonomic space which is more general than a Riemannian space and generalized the parallelism of Levi-Civita and geodesic curves in that space. From another standpoint $Z$. Horak") considered a non-holonomic region as a space with a non-holonomic dynamical system. These spaces were afterwards discussed by several authors. The non-holonomic system in a space of line-elements with an affine connection was first discussed by T. Hosokawa ${ }^{3}$.

We now suppose that at each point $x$ of an $n$-dimensional space $n$ independent differential forms

$$
d s^{a}=A^{\alpha}\left(x^{\alpha}, d x^{\beta}\right) \quad\left(\begin{array}{rl}
\alpha, \beta & =1,2, \cdots, n ; \\
a & =1,2, \cdots, n
\end{array}\right)
$$

are given for a displacement $d x$, where $A^{a}(x, d x)$ are homogeneous of degree one in the $d x$. If we write $(0.1)$ in the form

$$
d \mathbf{s}^{a}=\lambda_{\alpha}(x, d x) d x^{\alpha} \quad\left(\lambda_{\alpha}^{a}(x, d x)=\frac{\partial A^{a}}{\partial\left(d x^{\alpha}\right)}\right),
$$

$\lambda_{\alpha}^{a}(x, d x)$ depend on the direction of $d x$ only and have a non-vanishing determinant. As easily seen, $\lambda_{\alpha}^{\alpha}$ is covariant in $\alpha$. Hence we can define in the space of line-elements $\left(x, x^{\prime}\right)$ a special non-holonomic ${ }^{4)}$ system by

$$
d s^{a}=\lambda_{\alpha}^{\prime}\left(x, x^{\prime}\right) d x^{\alpha}
$$

which determines the displacement of a point in this system. $d s^{x}$ coincides with the original $A^{n}(x, d x)$ when and only when the displacement lies in the direction of the line-element:

$$
\text { (n.3b) } \quad s^{\prime \alpha}=\lambda_{\alpha}^{\alpha}\left(x, x^{\prime}\right) x^{\prime \alpha}=A^{a}\left(x, x^{\prime}\right) \text {. }
$$

In this paper we treat such non-holonomic systems and derive some fundamental quantities. We find that by use of our system the well known Cartan connection of a Finsler-space can be- expressed far more neatly than by general non-holonomic systems.

The author wishes to express her sincere thanks to Prof.A. Kawaguchi for his kind guidance.

\footnotetext{
1) G. VRanceanu, Sur les espaces non holonomes; Sur le calcul différentiel abs,lu pur les variétés non loolon'mes, C.R., 183(1926), p. 852 and 1083.

2) Z. Honak, Sur une générulištion de la notion de variété, Publ. Fac. Sc. Univ. Masaryk, Brno, 86 (1927), pp. 1-20.

3) T. Hosokawa, Ueber niclut-lh,lonome Uebertragung in allgemeiner Mannigfaltigkeit $T_{n}$, Jour. Fac. Sci. Hokkaido Imp. Univ., I, 2(1934), pp. 1-11.

4) We use Greek indices in holonomic systems and Latin indices in nom-holonomic systems.
} 
1 1. Fundamental properties of non-holonomic systems. From the given field of the $n$ independent covariant vectors $\lambda_{\alpha}^{a}\left(x, x^{\prime}\right)$ we first derive that of the reciprocal contravariant vactors $\lambda_{t}^{\alpha}\left(x, x^{\prime}\right)$ as follows. If we solve the equations $(0.1)$ for $d x^{\alpha}$, the solutions may be of the form

$$
d x^{\alpha}=B^{\alpha}(x, d s)
$$

with homogeneous function $B^{\alpha}$ of degree one; we then put

$$
\frac{\partial B^{\alpha}}{\partial\left(d s^{a}\right)}=\lambda_{l b}^{\alpha}(x, d s) \quad \text { or } \quad=\lambda_{a}^{\alpha}(x, d x) .
$$

(In general, when we substitute $d s$ in $f(x, d s)$ by $(0.1)$ we write $f(x, d x)$ and vice versa; further, in case $x^{\prime}$ replaces $d x$ we write $s^{\prime}$ in place of $d s$. ) If we differentiate (1.1) with respect to $d x^{\beta}$, we get

$$
\lambda_{a l}^{\alpha}\left(x, x^{\prime}\right) \lambda_{\beta}^{\prime \prime}\left(x, x^{\prime}\right)=\delta_{\beta}^{\alpha} \text {. }
$$

Similarly differentiating $(0.1)$ we get

$$
\lambda_{a}^{\prime \prime}\left(x, x^{\prime}\right) \lambda_{b}^{\alpha}\left(x, x^{\prime}\right)=\delta_{b}^{\prime \prime} .
$$

(1.3) and (1.4) show the reciprocity of the two fields $\lambda_{\alpha}^{a}$ and $\lambda_{t !}^{\alpha} \cdot$ we write also by the above convention

$$
\text { (1.5) } \quad \lambda_{i l}^{\alpha}\left(x, s^{\prime}\right){ }^{\prime \prime} \lambda_{\beta}^{a}\left(x, s^{\prime}\right)=\delta_{\beta}^{\alpha}, \quad{ }^{*} \lambda_{\alpha}^{a}\left(x, s^{\prime}\right){ }^{*} \lambda_{l,}^{\alpha}\left(x, s^{\prime}\right)=\delta_{b}^{t} \text {. }
$$

We now introduce the following fundamental operation formally called the partial differentiation

$$
\frac{\partial^{*} f}{\partial s^{i}}=\frac{\partial^{*} f}{\partial x^{\alpha}} \lambda^{\alpha}=\left(\frac{\partial f}{\partial x^{\alpha}}+\frac{\partial f}{\partial x^{\prime \beta}} \frac{\partial^{*} \lambda^{\beta}}{\partial x^{\alpha}} s^{\prime \prime}\right){ }^{*} \lambda_{t b}^{\alpha} ;
$$

the differentiation symbol $\partial / \partial s^{a}$ has only a formal meaning. We must note the obvious fact that $\partial f / \partial x^{\alpha}$ and $\partial " f / \partial x^{\alpha}$ are differerent:

$$
\frac{\partial f}{\partial x^{\alpha}}=\frac{\partial^{*} f}{\partial x^{\alpha}}+\frac{\partial^{\prime \prime} f}{\partial s^{\prime \prime}} \frac{\partial \lambda_{s}^{\prime \prime}}{\partial x^{\alpha}} x^{\prime \beta} \text {. }
$$

Applying $\partial / \partial s^{\prime \prime}$ on (1.6) and permuting the indices $a$ and $b$, we easily obtain

$$
\partial^{\prime \prime} f s^{\prime \prime}-\frac{\partial^{2} f}{\partial s^{3} \partial s^{u}}=-\omega_{i b}^{\prime} \frac{\partial^{*} f}{\partial s^{c}}
$$

where

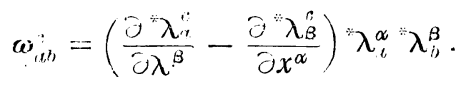

On the other hand, since

we get

$$
\begin{aligned}
& \frac{\partial^{*} f\left(x, s^{\prime}\right)}{\partial s^{\prime \iota}}=\frac{\partial f\left(x, x^{\prime}\right)}{\partial x^{\prime \alpha}} \lambda_{\iota}^{\alpha}, \\
& \frac{\partial^{2 *} f\left(x, \boldsymbol{s}^{\prime}\right)}{\partial \boldsymbol{s}^{\prime \prime \partial} \boldsymbol{s}^{\prime \prime}}=\frac{\partial^{2} f\left(x, x^{\prime}\right)}{\partial x^{\prime \alpha} \partial x^{\prime \beta}} \lambda_{i t}^{\alpha} \lambda^{\beta}+\frac{\partial f}{\partial x^{\prime \alpha}} \frac{\partial^{\prime \prime} \lambda^{\alpha}}{\partial \boldsymbol{s}^{\prime \prime}},
\end{aligned}
$$

$$
\frac{\partial^{2 *} f\left(x, s^{\prime}\right)}{\partial \boldsymbol{s}^{\prime a} \partial \boldsymbol{s}^{\prime},}-\frac{\partial^{2} f\left(x, x^{\prime}\right)}{\partial x^{\alpha} \partial x^{\prime \beta}} \lambda_{b}^{\alpha} \lambda^{\beta}=\Omega_{a b}^{c} \frac{\partial^{*} f}{\partial \boldsymbol{s}^{\prime c}}
$$

where

$$
\Omega_{n b}=\frac{\partial \lambda^{\alpha}}{\partial s^{\prime \prime}} \lambda_{x}^{c}
$$


The quantities $\omega_{a b}^{c}$ and $\Omega_{a b}^{c}$ will play an important rôle in the non-holonomic system (0.3).

We shall proceed to find the relations between these quantities $\omega_{b c}^{x}$ 's and $\Omega$; 's under transformations of non-holonomic system. If two systems are determined by the functions $A^{a}$ 's and ' $A^{i}$ 's, then we have

$$
s^{\prime i}={ }^{\prime} A^{i}\left(x, x^{\prime}\right), \quad d^{\prime} s^{i}=\lambda_{\alpha}^{i}\left(x, x^{\prime}\right) d x^{\alpha} \text {. }
$$

By. $(0.3 \mathrm{~b})$, (1.12) $s^{\prime i}$ will directly be transformed into ' $s^{\prime a}$ by the equations of the form

$$
s^{\prime \prime}=C^{a}\left(x,{ }^{\prime}{ }^{\prime}\right), \quad A^{a}=C^{\prime \prime}\left(x, A^{i}\right)
$$

where $C^{\prime \prime}$ are mutually independent in ' $\mathrm{s}^{\prime i}$ and homogeneous of degree one. From (0.1), (1.12), (1.13) we obtain by differentiation

$$
\lambda_{\alpha}^{a}=C_{i}^{\alpha} \lambda_{\alpha}^{i}, \quad ' \lambda_{i}^{\alpha}=C_{i}^{\prime} \lambda_{x}^{\alpha},
$$

where

$$
C_{i}^{x}=\frac{\partial C^{a}}{\partial^{\prime} s^{\prime}}
$$

Hence $d s^{a}$ are transformed as

$$
d s^{a}=C_{i}^{a}\left(x, s^{\prime}\right) d^{\prime} s^{i}
$$

this is nothing but the non-holonomic transformation of our systems. The inverse equations of (1.13), (1.14), (1.15) run as
${ }^{\prime} A^{i}={ }^{\prime} C^{\prime}\left(x, A^{\alpha}\right)$,$$
{ }^{\prime} \lambda_{\alpha}^{\prime}={ }^{\prime} C_{t}^{i} \lambda_{\alpha}^{\prime \prime}
$$

$$
\lambda_{a}^{\alpha}={ }^{\prime} C_{a}^{j{ }^{\prime}} \lambda_{j}^{\alpha}
$$$$
\left({ }^{\prime} C_{a}^{j}=\frac{\partial^{\prime} C^{j}\left(x, s^{\prime}\right)}{\partial s^{\prime a}}\right),
$$

$$
d^{\prime} s^{i}={ }^{\prime} C^{i} d s^{\prime}
$$

Obviously we have

$$
{ }^{\prime} C_{a}^{i} C_{i}^{\prime}=\delta_{a}^{\prime}, \quad{ }^{\prime} C_{a}^{i} C_{j}^{n}=\delta_{j}^{i} .
$$

Now, differentiating the second equation of (1.14) with respect to ' $s$ 's and noticing the homogeneity of $C_{k}^{\prime}$ we get the transformation formula of the quantity $\Omega_{a b b}^{c}$ in the form

$$
{ }^{\prime} \Omega_{i j}^{k}=\frac{\partial C_{i}^{\gamma}}{\partial \boldsymbol{s}^{\prime j}} \boldsymbol{C}_{a}^{k}+C_{i}^{a} \boldsymbol{C}_{j}^{\prime \prime} C_{c}^{k} \Omega_{a b .}^{c}
$$

On the other hand, in virtue of $\left(1.14^{\prime}\right)$ we see the quantity $\omega_{a b}^{c}$ is transformed as follows

$$
\begin{aligned}
& { }^{\prime} \boldsymbol{\omega}_{i j}^{k}=C_{i}^{\prime} \boldsymbol{C}_{j}^{b}\left\{\left(\frac{\partial^{* \prime} C_{i t}^{k}}{\partial \boldsymbol{S}^{\prime \prime}}-\frac{\partial^{* \prime} C_{b}^{k}}{\partial \boldsymbol{s}^{a}}\right)+\boldsymbol{C}_{c}^{k} \boldsymbol{\omega}_{a b}^{c}\right\}
\end{aligned}
$$

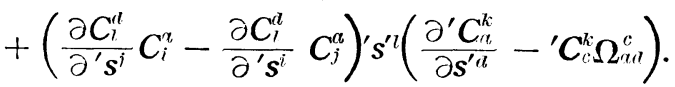

Next we shall derive the covariant derivative of tensors with respect to $s^{\prime}$ in our non-holonomic systems. The components of a vector $v^{\alpha}$ or a

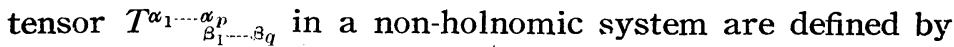

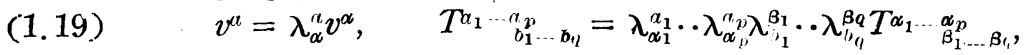

where $v^{\alpha}$ and $T^{\alpha_{1} \ldots} \beta_{1} \ldots .$. are components in the holonomic coordinate system $x$. In holonomic systems the partial derivatives of a vector $v^{\alpha}(x, x)$ with 
respect to $x^{\prime}$ are components of a tensor of degree two, but in a nonholonomic system the partial derivatives are not generally those of a tensor. Nevertheless the modified derivatives

$$
v^{\prime \prime}: b=\frac{\partial^{*} v^{a}}{\partial \mathbf{s}^{\prime \prime}}+\Omega_{i l b}^{a} v^{\prime \prime}={ }^{*} v_{; b}^{a}+\Omega_{(l)}^{\prime} v^{a}
$$

are components of a tensor of degree two, where "; denotes partial differentiation with respect to $s^{\prime}$. This can be easily seen in help of (1.17) or by the following. Indeed (1.20) are written by the definition of $\Omega_{b c}^{c}$ as

$$
v_{: b b}^{a}=\lambda_{\alpha}^{a} \lambda_{b}^{\beta} \frac{\partial v^{\alpha}}{\partial x^{\prime \beta}} .
$$

By this reason, we call the tensor $v^{a}: b$ the covariant derivative of the vector field $v^{a}$ with respect to $s^{\prime}$.

Generally for components of a tensor field $T^{a 1 \ldots a_{p}} \underset{b_{1} \ldots b q}{a,}$, we consider likewise

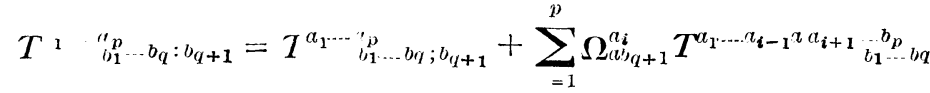

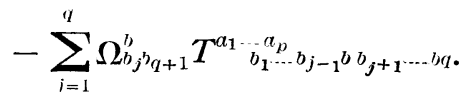

It can be shown that $T^{a_{1} \ldots a_{1} b_{1} \ldots h_{q}: b_{q+1}}, \mathrm{~s}$ are non-holonomic components of the ordinary covariant derivative with respect to $x^{\prime}$, that is

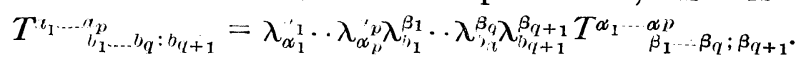

Accordingly we call them the covariant derivative of the tensor field $T$ with respect fo $s^{\prime}$.

2. Fundamental quantities in the Finsler space. The arc length of a curve $x^{\alpha}=x^{\alpha}(t)$ in a Finsler space is given by an integral

$$
s=\int \mathfrak{2}\left(x, x^{\prime}\right) d t
$$

where $2\left(x, x^{\prime}\right)$ is homogeneous of degree one in the $x^{\prime}$. To introduce an invariant connection we usually consider the manifold of line-elements $\left(x, x^{\prime}\right)$, each of which is composed of a point $x$ and a direction $x^{\prime}$ in this point. As is well known, E. Cartan established the euclidean connection by setting four postulates ${ }^{5)}$. This is the space with which we shall concern here.

In the Finsler space we consider a non-holonomic system defined by (0.3) and represent $\mathscr{L}\left(x, x^{\prime}\right)$ in $x$ and $s^{\prime}$ :

$$
\mathbb{L}\left(x, x^{\prime}\right)=* \mathbb{R}\left(x, s^{\prime}\right)
$$

which is homogeneous of degree one in the $s^{\prime}$. By differentiation we get

$$
\frac{\partial\left(x, s^{\prime}\right)}{\partial s^{\prime \prime}}=\frac{\partial \mathcal{L}\left(x, x^{\prime}\right)}{\partial x^{\alpha}} \lambda_{a}^{\alpha} \text {. }
$$

Putting the vector

\footnotetext{
5. L. Cantan, Les espres de Finsler, Actualités sci. et ind., 79 (1934).
} 
we obtain

$$
l_{x}=\frac{\partial \mathfrak{Q}}{\partial x^{\prime \alpha}}
$$

$$
l_{x}=\frac{\partial * Q}{\partial s^{\prime(b)}}
$$

If we put

$$
\mathfrak{F}=\frac{1}{2} \mathfrak{L}^{2} \text { and } g_{\alpha \beta}=\frac{\partial^{2} \mathfrak{F}}{\partial x^{\prime \alpha} \partial x^{\prime \beta}}
$$

the tensor $g_{\alpha \beta}$ serves as the fundamental metric tensor in the manifold of line-elements. By virtue of (2.3) we see that

$$
\begin{aligned}
& \mathfrak{F}={ }^{*} \mathfrak{w}, \quad *{ }_{\mathfrak{F} ;}=\lambda_{x}^{\alpha} \frac{\partial \tilde{F}}{\partial x^{\prime \alpha}},
\end{aligned}
$$

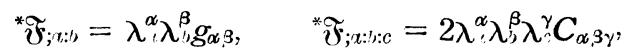

where we put $C_{\alpha \beta \gamma}=\frac{1}{2} \partial g_{x, \beta} / \partial x^{\prime \gamma}$ following Cartan. Thus the metric tensor $g_{x \beta}$ and $C_{\alpha \beta \gamma}$ are given in the non-holonomic system by

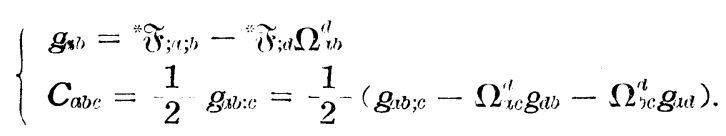

As can be seen by (2.5), these components are symmetric in their indices and it holds

$$
C_{a b c} s^{c}=0 .
$$

3. Parameters of connection. In our non-holonomic system we can introduce the covariant differential of a contravariant vector field $v$ in the form

and put

$$
\delta v^{\gamma}=\lambda_{\alpha}^{\gamma} \delta v^{\alpha}=\lambda_{\alpha}^{\prime}\left(d v^{\alpha}+\Gamma_{\beta \gamma}^{\alpha \alpha} v^{\beta} d x^{\gamma}+C_{\beta \gamma}^{\alpha} v^{\beta} \delta x^{\prime \gamma}\right)
$$

If we put $\Gamma_{\beta \gamma}^{* \alpha} x^{\prime \beta} x^{\prime \gamma}=2 G^{\alpha}, \quad \delta x^{\prime \alpha}=d x^{\prime \alpha}+\left(\partial G^{\alpha} / \partial x^{\prime \gamma}\right) d x^{\gamma}$ following Cartan, then we have at once

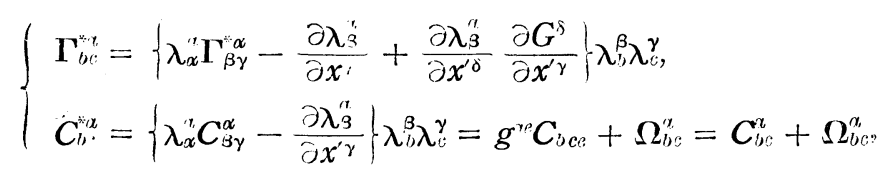

where $g^{(\eta)}$ is the contravariant tensor defined by $g_{a b}$ as usual: $g_{a j} g^{\tau}=\delta_{b}^{c}$. Further we get by (3.3)

$$
G_{b}^{x}=\Gamma_{c l}^{* a} \boldsymbol{s}^{\prime c}=\lambda_{\alpha}^{\alpha} \lambda^{\beta}, \frac{\partial G^{\alpha}}{\partial x^{\prime \beta}}-\frac{\partial \lambda_{\beta}^{\prime \prime}}{\partial x^{\gamma}} x^{\prime \beta} \lambda^{\gamma} .
$$

In holonomic systems the parameters of the euclidean connection of Cartan are given by the following formulas:

$$
\gamma_{\alpha \beta}^{\gamma}=\frac{1}{2}-g^{i \gamma}\left(\frac{\partial g_{x \delta}}{\partial x^{j}}+\frac{\partial g_{3 \delta}}{\partial x^{x}}-\frac{\partial g_{\alpha \beta}}{\partial x^{j}}\right)
$$




$$
\begin{aligned}
& G^{\gamma}={ }^{1}-\gamma_{\alpha \beta}^{\gamma} x^{\prime \alpha} x^{\prime \beta}, \\
& \Gamma_{\alpha \beta}^{\alpha_{\alpha} \gamma}=\gamma_{\alpha \beta}^{\gamma}+\left(C_{\alpha \beta \delta} \frac{\partial G^{\delta}}{\partial x^{\prime \epsilon}}-C_{\alpha \varepsilon \delta} \frac{\partial G^{\delta}}{\partial x^{\beta}}-C_{\beta \varepsilon \delta} \frac{\partial G^{\delta}}{\partial x^{\alpha}}\right) g^{\gamma \epsilon} .
\end{aligned}
$$

The parameters $C_{b r}^{* \prime}$ are determined already by (3.3). We shall write in the following the parameters $\Gamma_{a b}^{*}$ in terms of the fundamental quantities

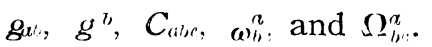

Differentiating $g_{\alpha \beta}=g_{m b} \lambda_{\alpha} \lambda_{\beta}^{b}$ and using (1.5), (1.11), (2.6), we get

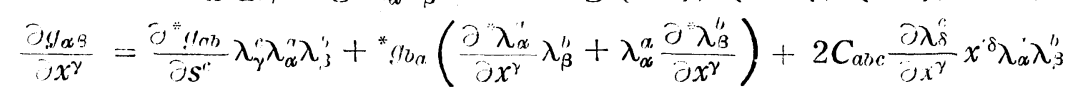

and consequently

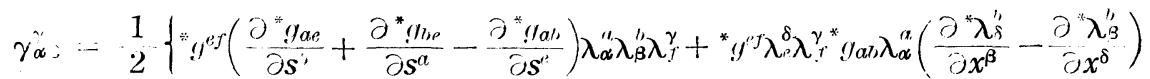

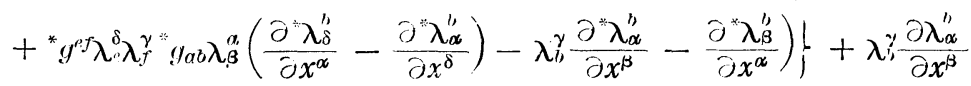

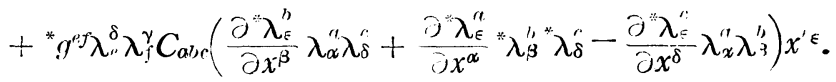

Noticing $\frac{\partial \lambda_{\alpha}^{r}}{\partial x^{s}} x^{\alpha}=\frac{\partial \lambda_{\alpha}^{f}}{\partial x^{s}} x^{\alpha}$ we get further

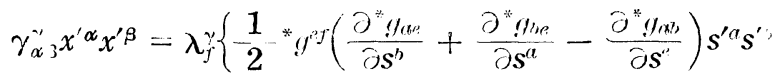

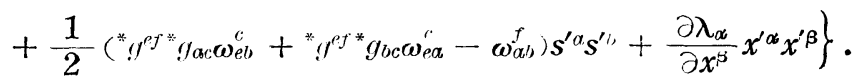

On the other hand we have from the first equations of (3.3)

$$
\Gamma_{, c}^{a} s^{\prime \prime} s^{\prime c}=\lambda_{x} \gamma_{\beta \gamma}^{\alpha} r^{\prime \prime} x^{\gamma}-\frac{\partial \lambda_{\alpha}^{a}}{\partial x^{\beta}} x^{\prime \beta} x^{\prime \alpha}
$$

From the two last equations we arrive at

$$
\Gamma_{l, c}^{* \prime c} s^{\prime \prime} s^{\prime e}=\gamma_{l, c}^{n} s^{\prime b} s^{\prime c}
$$

where we put

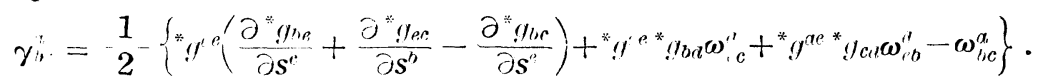

These correspond to the Christoffel's symbols $\left\{\begin{array}{c}\alpha \\ \beta_{\gamma}\end{array}\right\}$.

From (2.5), (2.6), (3.4) we obtain

$$
g^{\prime \gamma \delta}\left(C_{\alpha \beta \varepsilon} \frac{\partial G^{\varepsilon}}{\partial x^{\prime \delta}}\right)=\lambda_{e}^{\gamma} \lambda_{\alpha}^{\prime} \lambda_{\beta}^{\prime \prime *} !^{\rho j} C_{a b !}\left(G_{f}^{g}-\frac{\partial^{*} \lambda_{\Sigma}^{g}}{\partial x^{\delta}} x^{\prime \varepsilon} \lambda_{f}^{\delta}\right),
$$

by virtue of which we can derive from (3.5), (3.6)

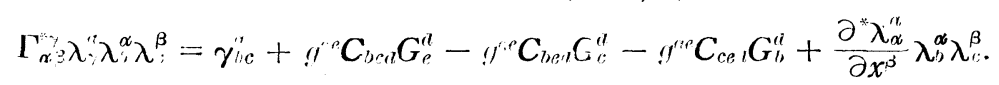

Using (1.6), (3.4) we calculate

$$
\left(\frac{\partial \lambda_{c}^{*}}{\partial x^{\prime \prime}} \frac{\partial G^{\delta}}{\partial x^{\prime}}-\frac{\partial \lambda_{\beta}^{\prime \prime}}{\partial x^{\gamma}}\right) \lambda_{b}^{\beta} \lambda_{c}^{\gamma}=\frac{\partial^{*} \lambda_{o}^{\prime \prime}}{\partial s^{\prime \prime \prime}} G_{c}^{d} \lambda_{b}^{\beta}-\frac{\partial^{*} \lambda_{\beta}^{\prime \prime}}{\partial x^{\delta}} \lambda_{b}^{\beta} \lambda_{c}^{\delta}=-\Omega_{b d}^{a} G_{c}^{d}-\frac{\partial^{*} \lambda_{\beta}^{\prime \prime}}{\partial x^{\gamma}} \lambda_{b}^{\beta} \lambda_{c}^{\gamma} .
$$

From these equations and (3.3) we get finally 


$$
\left.\Gamma_{b c}^{*, e}=\gamma_{b ;}^{\prime \prime}+{ }^{*}\right)^{a l l}\left(C_{b c e} G_{a}^{e}-C_{c i l e} G_{b}^{e}-C_{b d e} G_{c}^{e}\right)-\Omega_{b d}^{a} G_{c}^{d} .
$$

However $G_{a l}^{e}=\Gamma_{f i l}^{* e} s^{\prime f}$, hence we must find the formula of $G_{d b}^{\prime}$ written in terms of the fundamental quantities. This can be easily done.

From (3.10) we have successively

$$
\begin{aligned}
& G_{c}^{a}=\gamma_{b,}^{a} s^{\prime b}-{ }^{*} g^{(\prime \prime} C_{c l l} G_{b}^{\prime} s^{\prime \prime)}, \\
& G_{b}^{a} s^{\prime c}=\gamma_{b c}^{a} s^{\prime \prime} s^{\prime c}=2 G^{a} .
\end{aligned}
$$

and hence

$$
G_{c}^{a}=\gamma_{b c}^{n} s^{\prime \prime}-2^{*} g^{a \prime \prime} C_{c, l B} G^{e} .
$$

Thus the formulas: the second of (3.3), (3.9)-(3.12) completely determine the parameters of connection of Cartan in our non-holonomic system. We remark, however, the fact that $G^{a} \mathbf{s}^{\prime \prime}=G_{b}^{a}$ but $G_{b}^{x}$ does not coincide with $\partial G^{a} / \partial s^{\prime \prime}$ in general, and seek their relation. From the first of (3.3) we have

$$
G^{\alpha}=\lambda_{a}^{a}\left\{G^{a}+\frac{1}{2} \frac{\partial \lambda_{\gamma}^{a}}{\partial x^{\beta}} x^{\prime \gamma} x^{\prime \beta}\right\},
$$

and, differentiating this,

$$
\frac{\partial G^{\alpha}}{\partial x^{\prime \gamma}}=\lambda_{\tau}^{\alpha} \lambda_{\gamma}^{c} \frac{\partial G^{a}}{\partial s^{\prime c}}+\frac{1}{2}\left(\frac{\partial^{*} \lambda_{\gamma}^{a}}{\partial x^{j}}-\frac{\partial^{*} \lambda_{\delta}^{a}}{\partial x^{\gamma}}\right) x^{\prime \delta} \lambda_{t}^{\alpha}+\frac{\partial \lambda_{1}^{\alpha}}{\partial x^{\prime \gamma}} G^{a}+\lambda_{t}^{\alpha} \frac{\partial \lambda_{\delta}^{\prime \prime}}{\partial x^{\gamma}} x^{\prime \delta} .
$$

Hence we obtain from (3.4), (3.14)

$$
G_{b}^{a}=\frac{\partial G^{a}}{\partial s^{\prime j}}+\frac{1}{2} \omega_{b c}^{a} s^{c}+\Omega_{c h}^{a} G^{c}
$$

By use of this formula the connection parameters (3.10) may be written in the form

$$
\begin{aligned}
& \Gamma_{b c}^{* a}=\gamma_{b c}^{a}+g^{a l}\left(C_{b c e} \frac{\partial G^{e}}{\partial \boldsymbol{s}^{\prime l}}-C_{c b e} \frac{\partial G^{e}}{\partial \boldsymbol{s}^{\prime \prime)}}-C_{b l l e} \frac{\partial G^{e}}{\partial \mathbf{s}^{\prime c}}\right)-\Omega_{b l l}^{a} \frac{\partial G^{\prime b}}{\partial \boldsymbol{s}^{\prime c}} \\
& +\frac{1}{2}\left\{g^{a l l}\left(C_{c b e} \omega_{l f f}^{o}-C_{c l b} \omega_{b f}^{e}-C_{b l b c} \omega_{c \cdot f}^{e}\right)-\Omega_{l, l}^{x} \omega_{c \cdot f}^{\prime}\right\} s^{\prime f}
\end{aligned}
$$

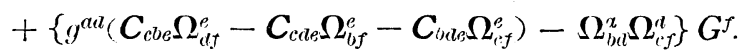

At last we notice that the extremal curves are given in the nonholonomic system by the equations

$$
\frac{d^{2} s^{\prime \prime}}{d s^{\prime}}+2 G^{a}\left(x, \frac{d s^{a}}{d s}\right)=0 .
$$

4. Curvature and torsion tensors. If we denote the basis vectors of the natural reference system defined at each line-element $\left(x, x^{\prime}\right)$ by $e_{a}$ $(\alpha=1, \cdots, n)$, we have the displacement of the centre $M(x)$

$$
d M=d x^{\alpha} e_{\alpha}
$$

which can be written in a non-holonomic system as

$$
\begin{gathered}
e_{a}=\lambda_{l}^{\alpha} e_{\alpha}, \\
d M=d s^{n} \lambda_{t}^{\alpha} e_{\alpha}=d s^{n} e_{a} .
\end{gathered}
$$

The $n$ vectors $e_{a}(a=1, \ldots, n)$ are the basis vectors of the non-holonomic system. Using the symbol $\omega_{a l}^{e}=\Gamma_{a b}^{* e} d s^{\prime \prime}+C_{a b}^{* e} \delta s^{\prime \prime}, d e_{a}=\omega_{a}^{e} e_{e}$ and denoting 
two infinitesimal displacements with $d_{1}$ ano $d_{z}$, we have

$$
\text { (4.4) } \quad d_{2} d_{1} M-d_{1} d_{2} M=\left\langle d_{2} d_{1} s^{a}-d_{1} d_{2} s^{a}\right) e_{a}+\left(d_{1} s^{a} \omega_{a}^{e}-d_{2} s^{a} \omega_{a}^{e}\right) e_{e} \text {. }
$$

From the first equations of (0.3a) it follows that

$$
\text { (4.5) } \quad d_{2} d_{1} s^{a}-d_{1} d_{\leq} s^{a}=\omega_{b c}^{a} d_{1} s^{b} d_{2} s^{c}+2 \Omega_{{ }^{\prime}\left|{ }_{|l|}\right|}^{a} G_{c !}^{a} d_{1} s^{b} d_{2} s^{c}-\Omega_{b c}^{a}\left(\delta_{2} s^{\prime c} d_{1} s^{b}-\delta_{1} s^{\prime c} d_{2} s^{b}\right) \text {. }
$$

Thus, if we put

$$
d_{2} d_{1} M-d_{1} d_{2} M=\Omega^{n} e_{a}
$$

we have

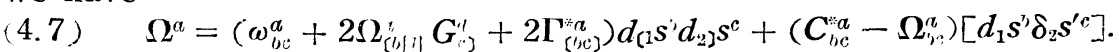

If our space is a general space of line-elements, not necessarily Finslerian but with an affine connection, we obtain the torsion tensors of two kinds as follows :
(4.8)
$T_{b c}^{a}=\omega_{b c}^{a}+2 \Omega_{(b, j)}^{a} G_{c]}^{f}+2 \Gamma_{[b c]}^{* a}$,
$" T_{b c}^{a}=C_{b c}^{* a}-\Omega_{b c}^{a}$

if the space is Finslerian, we get from (3.9), (3.10)

$$
2 \Gamma_{\{b, j]}^{* a}=-\omega_{b c}^{a}-2 \Omega_{(b, a l}^{a} G_{c,\}}^{l}
$$

and from the second equation of (3.3) $C_{l, c}^{* a}-\Omega_{b c}^{a}=C_{b c}^{b}$, hence

$$
\Omega^{a}=C_{b c}^{a}\left[d_{1} s^{b} \delta_{2} s^{\prime c}\right]
$$

$C_{m}^{a}$ being the only torsion tensor.

On the other hand, if we put the covariant differential $\delta v^{a}$ in the form

$$
\delta v^{a}=v_{\cdot b}^{a} d s^{b}+v_{\sharp b}^{a} \delta s^{\prime \prime}
$$

we get the covariant derivatives of two kinds by virtue of $(1.20),(3.2)$ :

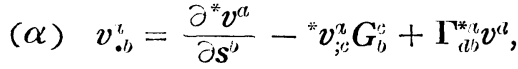

$$
\begin{aligned}
& \text { ( } \beta \text { ) } v_{: b}^{x}={ }^{*} v_{; b}^{a}+\Omega_{: b}^{a} v^{c} \text {. }
\end{aligned}
$$

As $C_{c b}^{* a}=\Omega_{c b}^{a}+C_{c b}^{a}$ and $C_{b b}^{t}$ is a tensor, we obtain from ( $\beta$ ) another covariant derivative

$$
v_{* b}^{a b}={ }^{*} v_{; b}^{i b}+C_{c b b}^{a b} v^{c} .
$$

We shall have therefore many tensors by combination of these derivatives.

After a complicated calculation, we get

$$
v_{\left\{\bullet b_{\bullet}\right]}^{\imath}=K_{a b c}^{a} v^{a}+{ }^{\prime} K_{b c}^{f} v_{r}^{\imath}
$$

where

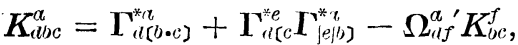

$$
\begin{aligned}
& { }^{\prime} K_{b c}^{f}=-G_{[b, c]}^{f}+G_{[b ; \mid q\}}^{f} G_{i}^{\prime \prime}+\Gamma_{[b c\}}^{* e} G^{f},
\end{aligned}
$$

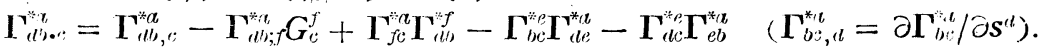

Further we have the following results

$$
v_{: l, c c}^{a}-v_{\bullet:: b}^{a}=L_{a l b c}^{a} v^{c}+{ }^{\prime} L_{b c}^{e} v_{: e,}^{a}
$$

where

$$
L_{c b c}^{a}=\Omega_{c i b c c}^{a}-\Gamma_{c c ; b}^{* a}+\Omega_{c b}^{c} \Gamma_{a e}^{* a}-\Omega_{c l}^{a} L_{b c}^{e}
$$

$$
\begin{aligned}
& { }^{\prime} L_{b c}^{e}=-\Gamma_{b c}^{* e}+G_{c ;, j}^{e}-\Omega_{c b}^{f} G_{f}^{\prime \prime} \text {, }
\end{aligned}
$$

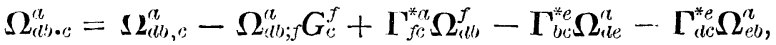


and

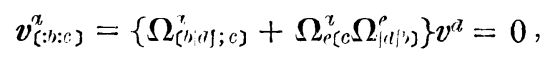

because

(4.19)

To the end we obtain

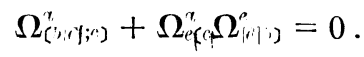

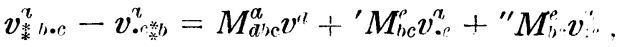

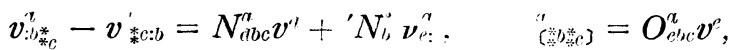

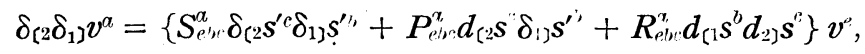

where

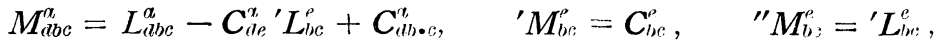

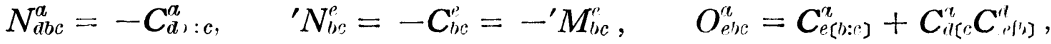

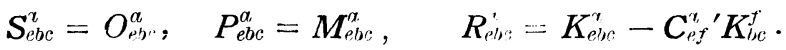

Such tensors can be also represented by the fundamental quantities $g_{x^{\prime}}$, $C_{a b c}, \omega_{b c}^{n}, \Omega_{b r}^{n}$ and their derivatives with respect to the $s$ and $s^{\prime}$.

REMARK. This paper was read at the meeting of the Mathematical Society of Japan in Nov., 1948. Recently the present author could read a paper of V. Wagner ${ }^{6)}$ sent to Prof. A. Kawaguchi, which, had many connections with mine and in some respects was more general. Especially $\mathrm{V}$. Wager considered $m$-dimensional non-holonomic referring manifolds. (March, 1949).

Mathematical. Instituty, dokkaido Univerity.

6) V. W AGNER, The inner geornetr, of non-linear non-liolonomic manifolds, Rec Math., N.S. 13 (1943), pp. 135-167. 